\title{
Effect of the Physicochemical Structure of Mudstone on Readsorption Behavior of Water
}

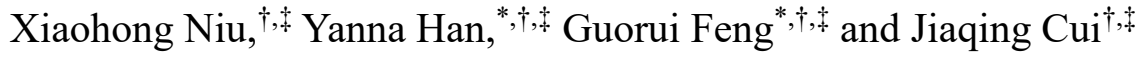

'College of Mining Engineering, Taiyuan University of Technology, Taiyuan 030024,

China

${ }^{\ddagger}$ Research Center of Green Mining Engineering Technology of Shanxi Province, Taiyuan 030024, China

*Email: hanyanna0504@163.com. Phone: +86 3516111086.

*Email: fguorui@163.com. Phone: +86 3516010177. 
Table S1. The Chemical Composition Analysis of Samples

\begin{tabular}{ccccccccccccccccc}
\hline & \multicolumn{11}{c}{ chemical composition (wt \%) } \\
\cline { 2 - 13 } & $\mathrm{SiO}_{2}$ & $\mathrm{Al}_{2} \mathrm{O}_{3}$ & $\mathrm{Fe}_{2} \mathrm{O}_{3}$ & $\mathrm{TiO}_{2}$ & $\mathrm{~K}_{2} \mathrm{O}$ & $\mathrm{SO}_{3}$ & $\mathrm{Na}_{2} \mathrm{O}$ & $\mathrm{MgO}$ & $\mathrm{CaO}$ & $\mathrm{P}_{2} \mathrm{O}_{5}$ & $\mathrm{ZrO}_{2}$ & $\mathrm{CeO}_{2}$ & $\mathrm{SrO}$ & $\mathrm{ZnO}$ & $\mathrm{MnO}$ & $\mathrm{BaO}$ \\
\hline $\mathrm{XT}$ & 57.8 & 37.0 & 1.33 & 0.98 & 0.94 & 0.84 & 0.35 & 0.32 & 0.15 & 0.07 & 0.07 & 0.06 & 0.02 & 0.01 & 0.01 & 0.00 \\
$\mathrm{SS}$ & 56.6 & 30.5 & 3.76 & 0.78 & 2.13 & 2.85 & 1.95 & 0.54 & 0.45 & 0.14 & 0.05 & 0.00 & 0.03 & 0.02 & 0.08 & 0.00 \\
$\mathrm{DN}$ & 58.7 & 33.4 & 1.53 & 0.98 & 3.04 & 0.25 & 0.74 & 0.94 & 0.15 & 0.11 & 0.03 & 0.00 & 0.02 & 0.01 & 0.00 & 0.07 \\
\hline
\end{tabular}




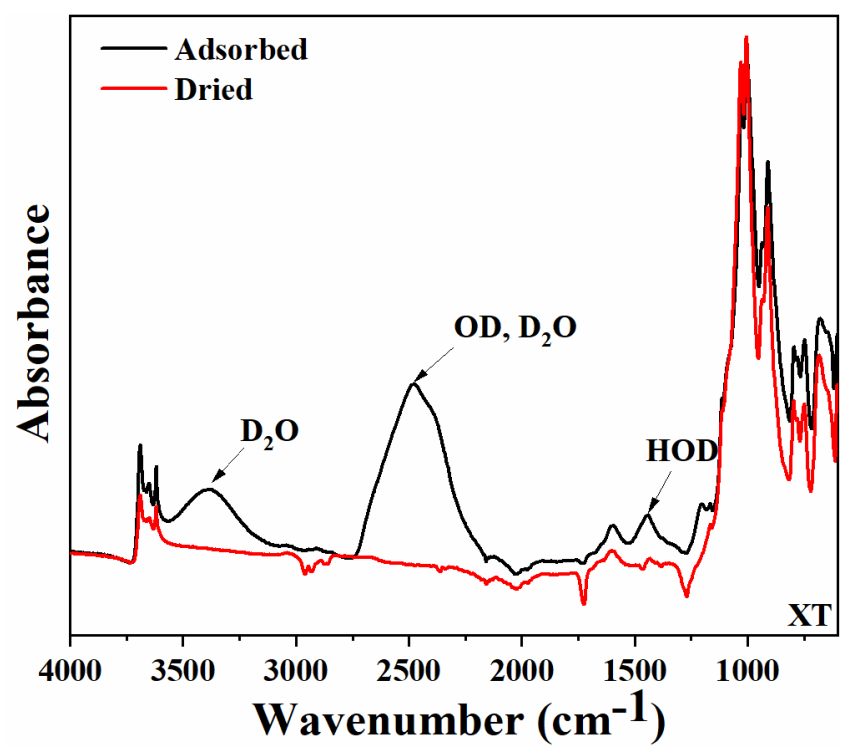

Figure S1. DRIFT spectra of dried XT and XT containing adsorbed $\mathrm{D}_{2} \mathrm{O}$ within the whole wavenumber measured at $23{ }^{\circ} \mathrm{C}$.

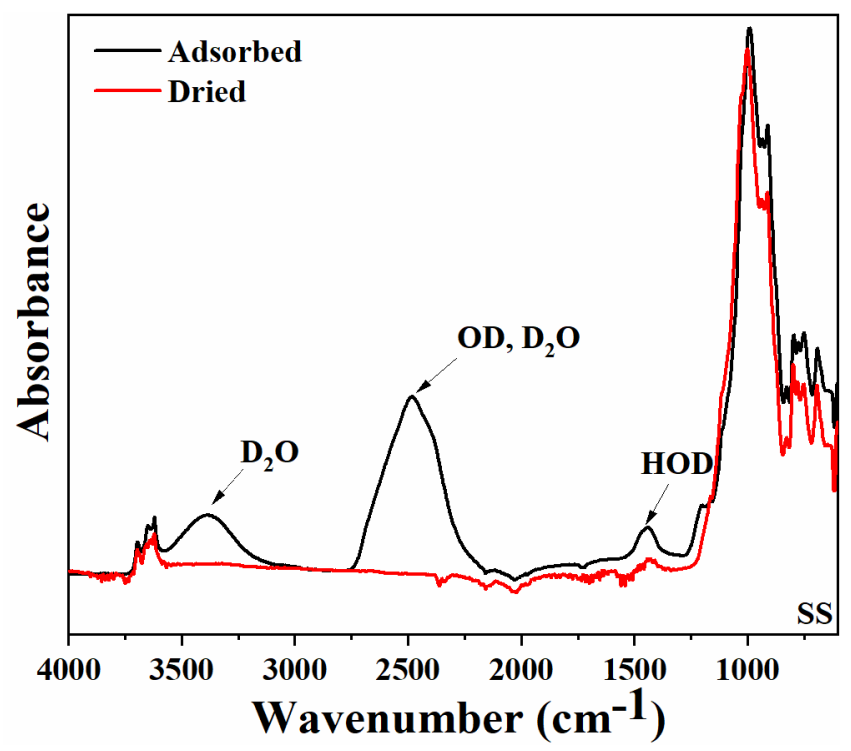

Figure S2. DRIFT spectra of dried SS and SS containing adsorbed $\mathrm{D}_{2} \mathrm{O}$ within the whole wavenumber measured at $23{ }^{\circ} \mathrm{C}$. 


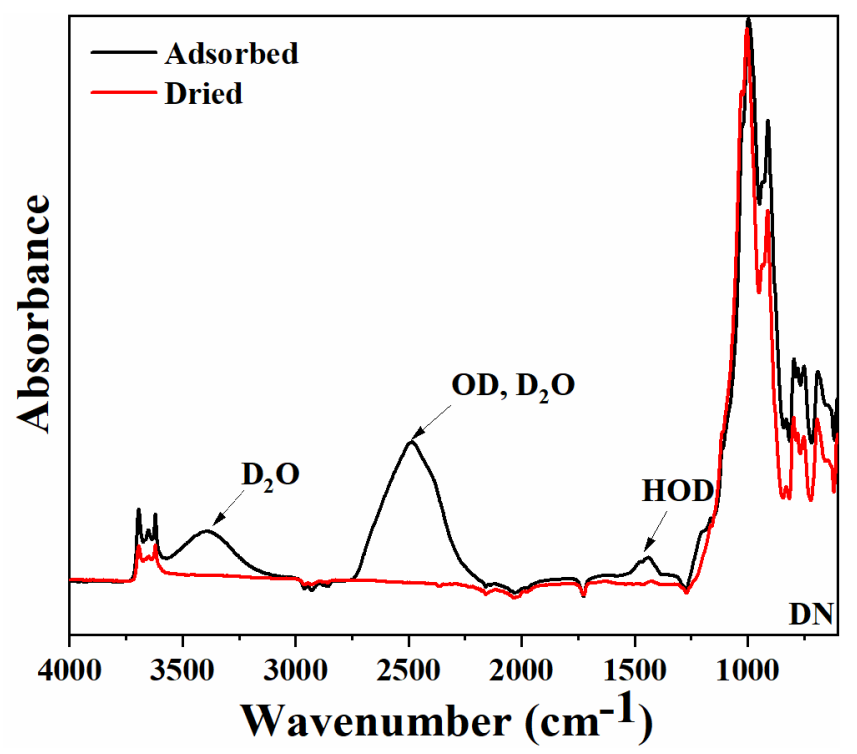

Figure S3. DRIFT spectra of dried DN and DN containing adsorbed $\mathrm{D}_{2} \mathrm{O}$ within the whole wavenumber measured at $23{ }^{\circ} \mathrm{C}$.



Figure S4. In situ DRIFT spectra of XT containing adsorbed $\mathrm{D}_{2} \mathrm{O}$ within the whole wavenumber. 


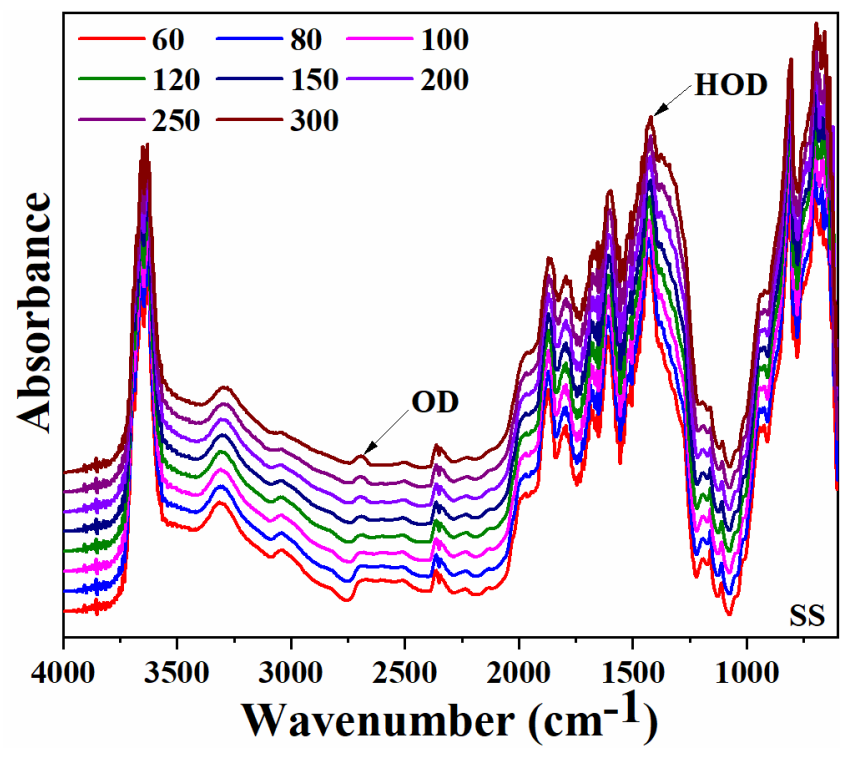

Figure S5. In situ DRIFT spectra of SS containing adsorbed $\mathrm{D}_{2} \mathrm{O}$ within the whole wavenumber.

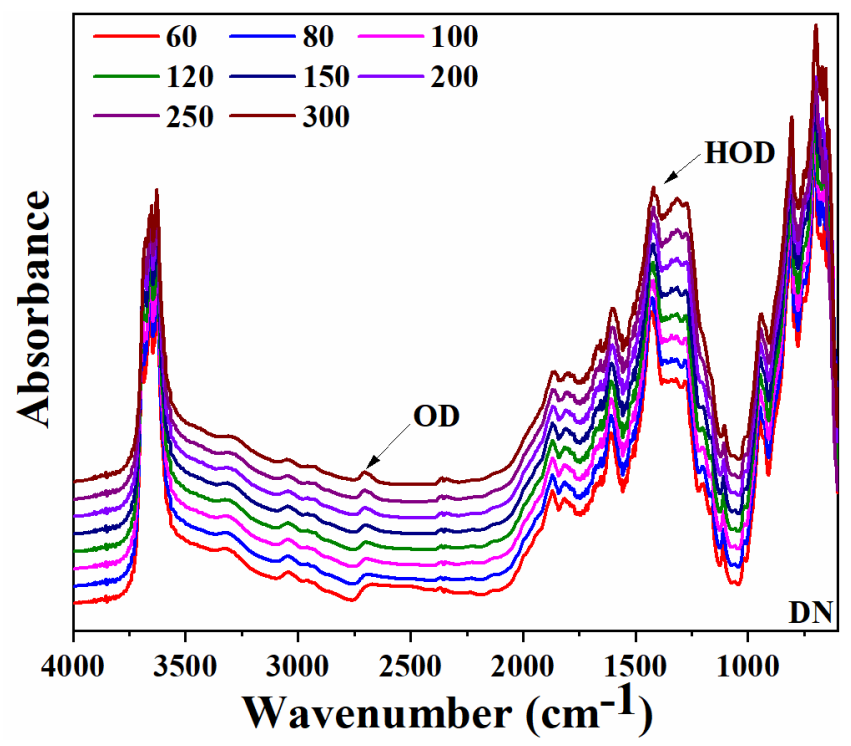

Figure S6. In situ DRIFT spectra of DN containing adsorbed $\mathrm{D}_{2} \mathrm{O}$ within the whole wavenumber. 